\title{
Evaluation of Genetic Variations in Organic Cationic Transporter 3 in Depressed and Nondepressed Subjects
}

\author{
Nina Hengen, Mitsi H. Lizer, and Robert S. Kidd \\ Bernard J. Dunn School of Pharmacy, Shenandoah University, 1775 North Sector Court, Winchester, VA 22601, USA \\ Correspondence should be addressed to Nina Hengen, nhengen@su.edu
}

Received 11 June 2011; Accepted 29 June 2011

Academic Editors: V. C. Abilio and P. S. D’Aquila

Copyright () 2011 Nina Hengen et al. This is an open access article distributed under the Creative Commons Attribution License, which permits unrestricted use, distribution, and reproduction in any medium, provided the original work is properly cited.

Organic cationic transporter 3 (OCT3, SLS22A3) has only recently emerged as one of the regulators of monoaminergic neurotransmission, which plays a critical role in the pathogenesis of depression and is a potential new antidepressant drug target. OCT3 single-nucleotide polymorphisms (SNPs) have been investigated for their association with psychiatric disorders such as methamphetamine use disorder and obsessive-compulsive disorder in children and adolescents, but not depression. This study was designed to evaluate the allele frequencies of seven OCT3 SNPs in a US Caucasian depressed population and compare these frequencies with a control group of nondepressed subjects. Informed consent and a DNA sample were obtained from 157 subjects and analysis was performed using real-time PCR. Allele and genotype frequencies were compared using a $t$-test and the Pearson chi-square analysis, respectively. There were no significant differences in OCT3 allele or genotype frequencies between the depressed and non-depressed groups for all seven SNPs evaluated.

\section{Introduction}

Depression affects up to $20 \%$ of the world population and is one of the top ten causes of morbidity and mortality worldwide [1]. Even though the precise neurobiological processes causing depression are not yet completely understood, it is certain that monoamine neurotransmission plays an important role [2], as the vast majority of currently available antidepressants alter serotonergic, noradrenergic, and/or dopaminergic neurotransmission [3].

Monoamine neurotransmission is regulated by the synaptic levels of neurotransmitters serotonin $(5-\mathrm{HT})$, norepinephrine (NE), and dopamine (DA). These levels are, in turn, determined by the activity of monoamine transporters [4]. Two uptake mechanisms are responsible for removal of synaptic monoamines. The high-affinity, low-capacity, substrate-specific, and $\mathrm{Na}^{+}$- and $\mathrm{Cl}^{-}$-dependent system consists of serotonin (SERT), norepinephrine (NET), and dopamine (DAT) transporters [4-6]. On the other hand, a low-affinity, high-capacity, multispecific, and $\mathrm{Na}^{+}$- and $\mathrm{Cl}^{-}$independent system includes organic cation transporters (OCT) 1, 2, and 3 (also known as solute carrier family 22 members 1-3, (SLC22A)1-3) [7]. All three OCTs are capable of transporting multiple endogenous and exogenous organic cations, including neurotransmitters 5-HT, NE, epinephrine, DA, and histamine [7].

OCTs show a broad tissue distribution, with expression in the CNS and peripheral tissues in both humans and rodents [7]. While all three OCT subtypes are expressed in the brain [7], OCT3 is preferentially expressed in brain regions that belong to monoamine pathways, such as raphe nuclei, striatum, ventral tegmental area, locus coeruleus, hippocampus, and cortex [8-10], as well as the dorsomedial hypothalamus which is the brain region involved in stress regulation of monoamine neurotransmission $[11,12]$. Considering the important role of stress in the pathogenesis of depression [13], it is interesting that OCT3 is more sensitive to inhibition by the stress hormone corticosterone than the other two OCTs $[12,14-16]$.

The fact that OCT3 transports 5-HT, NE, and DA [7] and is associated with monoamine pathways [8-10], points towards OCT3 playing a role in the regulation of monoamine neurotransmission. Indeed, OCT3-deficient mice show altered monoamine neurotransmission $[9,17]$, anxietyrelated behavior $[9,18]$, stress response [9], and response to psychostimulants [9]. Therefore, OCT3 could be a candidate 
gene for association with neuropsychiatric disorders, such as depression, where monoamine neurotransmission plays a critical role.

Studies investigating the association of different monoamine transporter gene polymorphisms with depression have primarily focused on SERT and NET gene polymorphisms [19-23]. However, the association between OCT3 gene polymorphisms and depression has not been examined so far. Recently, OCT3 gene single-nucleotide polymorphisms (SNPs) have been investigated for their association with several psychiatric and nonpsychiatric disorders, such as coronary artery disease [24], diabetic nephropathy, and hypertension [25]. Psychiatric disorders examined so far are methamphetamine use disorder [26] and obsessivecompulsive disorder in children and adolescents [27], but not depression. Therefore, the goal of this study was to evaluate seven OCT3 gene SNPs in a US Caucasian depressed population and compare these allele frequencies and resulting genotypes with a control group of nondepressed subjects.

\section{Methods}

2.1. Subjects. This cross-sectional study utilized subjects curr-ently being treated for depression at a communitybased psychiatric practice and an ambulatory care clinic (depressed group). The nondepressed control group was recruited from the same ambulatory care clinic and the community. Subjects in the depressed group were identified by either chart review or referred by their physician with inclusion criteria of a documented diagnosis of depression and no other Axis I or Axis II diagnosis. All self-reported as Caucasian. Patients under the age of 18 years old or with a concurrent diagnosis of bipolar disorder were excluded. Subjects in the nondepressed (control) group were selfreferred and reported no previous nonpharmacologic or pharmacologic treatment of depression or bipolar disorder. All self-reported as Caucasian. Axis II diagnosis in the control group was unknown. Subjects under the age of 18 years old were also excluded from the control group. No other medical comorbidity information was collected for either group. All subjects signed an informed consent, and two cheek swab samples were obtained for DNA collection. This study was approved by the Shenandoah University Institutional Review Board prior to its commencement.

2.2. Genotyping. Genomic DNA (gDNA) was isolated from the cheek swab samples using a Qiagen QiaCube automated DNA isolation instrument (Qiagen Inc, Chatsworth, Calif, USA) following the manufacturer's protocol and then frozen at $-20^{\circ} \mathrm{C}$ until the time of genotyping. A total of seven OCT3 gene SNPs were selected for this study: SNP1 (rs1810126), SNP2 (rs204832), SNP3 (rs2048327), SNP4 (rs665185), SNP5 (rs4709426), SNP6 (rs3106164), and SNP7 (rs 2292334). SNPs 3, 4, 5, 6, and 7 have been previously assessed for their correlation with methamphetamine use disorder in a Japanese population [26]. SNPs 1 and 7 have been found to be present in Caucasian population [28]. In addition, SNPs 1, 2, 3, and 7 have been found to be associated with reduced OCT3 mRNA levels in the human liver [29]. The genotyping was performed on an Applied Biosystems 7300 real-time PCR (Applied Biosystems, Foster City, Calif, USA) with commercially available, validated TaqMan SNP Genotyping Assays for OCT3 (Applied Biosystems, Foster City, CA). The genotyping assays used included nonlabeled primers and one wild-type and one variant allele-specific fluorescent TaqMan labeled oligonucleotide probes. PCR was performed with a reaction volume of $10 \mu \mathrm{L}$, which included $4.75 \mu \mathrm{L}$ of TaqMan Genotyping Master Mix (Applied Biosystems), $0.5 \mu \mathrm{L}$ of $20 \mathrm{x}$ SNP Genotyping Assay, $3.75 \mu \mathrm{L}$ of DEPC $\mathrm{H}_{2} \mathrm{O}$, and $1.0 \mu \mathrm{L}$ of gDNA. The PCR cycling conditions were as follows: 1 cycle of $50^{\circ} \mathrm{C}$ for 2 minutes, followed by 1 cycle of $95^{\circ} \mathrm{C}$ for 10 minutes, and 50 cycles of $92^{\circ} \mathrm{C}$ for 15 seconds and $60^{\circ} \mathrm{C}$ for 90 seconds. Allelic discrimination was evaluated by measuring endpoint fluorescence intensity. These results were analyzed with SDS software Version 1.3 (Applied Biosystems), and the corresponding genotypes were assigned.

2.3. Statistical Analysis. A power calculation was performed, and it was estimated that 72 subjects would be required in each group to detect a clinically significant difference of $20 \%$ in each allele frequency, with a power of $80 \%$ and a significance level of $5 \%$. Deviation from the Hardy-Weinberg equilibrium was tested by allele counting and evaluated by the chi-square $\left(\chi^{2}\right)$ test. Allele frequencies were first evaluated with Levene's test for equality of variances and then compared between the two groups using the Student $t$-test. Genotypes were compared between groups using the Pearson $\chi^{2}$ test. A $P<0.05$ was considered statistically significant. All statistical analyses were performed using PASW v18.0 (SPSS Inc. Chicago, Il, USA).

\section{Results}

A total of 157 subjects were recruited into the study. There were 82 subjects in the depressed group $(28.6 \%$ males and $71.4 \%$ females) and 75 subjects in the control group (37.8\% males and $62.2 \%$ females). The OCT3 genotype frequencies for all seven SNPs examined did not deviate from the Hardy-Weinberg equilibrium in either the depressed or nondepressed control group. Genotype and allele frequencies for those seven SNPs in depressed patients and nondepressed control subjects are summarized in Table 1. No significant difference was found in either genotype prevalence or allele frequencies between the depressed and nondepressed control groups (Table 1). In addition, this study found none of the SNPs evaluated to be in complete linkage disequilibrium with one another.

\section{Discussion}

To the authors' knowledge, this is the first study to examine the potential association of OCT3 gene polymorphisms with depression. We evaluated the allele frequencies of seven OCT3 gene SNPs in the US Caucasian patients actively being treated for depression and compared these frequencies with 
TABLE 1: Genotype and allele distributions of OCT3 SNPs in depressed and nondepressed subjects.

\begin{tabular}{|c|c|c|c|c|c|c|c|c|}
\hline \multirow{2}{*}{ SNP } & \multirow{2}{*}{ Sample } & \multicolumn{4}{|c|}{ Genotype } & \multicolumn{3}{|c|}{ Allele frequency } \\
\hline & & $\mathrm{M} / \mathrm{M}$ & $\mathrm{M} / \mathrm{m}$ & $\mathrm{m} / \mathrm{m}$ & $P$ value & M & $\mathrm{m}$ & $P$ value \\
\hline SNP1 & Depressed & 30 & 38 & 14 & \multirow{2}{*}{0.433} & 0.598 & 0.442 & \multirow{2}{*}{0.364} \\
\hline rs 1810126 & Control & 26 & 30 & 19 & & 0.547 & 0.453 & \\
\hline SNP2 & Depressed & 29 & 39 & 14 & \multirow{2}{*}{0.442} & 0.591 & 0.409 & \multirow{2}{*}{0.425} \\
\hline rs2048327 & Control & 26 & 30 & 19 & & 0.547 & 0.453 & \\
\hline SNP3 & Depressed & 29 & 40 & 13 & \multirow{2}{*}{0.163} & 0.598 & 0.402 & \multirow{2}{*}{0.207} \\
\hline rs3088442 & Control & 25 & 29 & 21 & & 0.527 & 0.473 & \\
\hline SNP4 & Depressed & 21 & 44 & 17 & \multirow{2}{*}{0.737} & 0.524 & 0.476 & \multirow{2}{*}{0.694} \\
\hline rs665185 & Control & 23 & 36 & 16 & & 0.547 & 0.453 & \\
\hline SNP5 & Depressed & 22 & 42 & 18 & \multirow{2}{*}{0.954} & 0.524 & 0.476 & \multirow{2}{*}{0.783} \\
\hline rs4709426 & Control & 21 & 39 & 15 & & 0.54 & 0.46 & \\
\hline SNP6 & Depressed & 29 & 37 & 16 & \multirow{2}{*}{0.791} & 0.579 & 0.421 & \multirow{2}{*}{0.916} \\
\hline rs3106164 & Control & 28 & 30 & 17 & & 0.573 & 0.427 & \\
\hline SNP7 & Depressed & 30 & 38 & 14 & \multirow{2}{*}{0.403} & 0.598 & 0.402 & \multirow{2}{*}{0.430} \\
\hline rs2292334 & Control & 27 & 29 & 19 & & 0.553 & 0.447 & \\
\hline
\end{tabular}

M: major allele; $\mathrm{m}$ : minor allele.

a control group of nondepressed subjects. No significant difference was found in allele frequencies between the depressed and nondepressed groups.

SNPs 3, 5, and 6 have been found to be associated with methamphetamine dependence disorder in a Japanese population [26]. That study found the major (M) allele frequency in the control group to be $50.9 \%$ for SNP 3, $59.8 \%$ for SNP 5, and $69.1 \%$ for SNP 6. In our study, M allele frequencies in the control group were $59.8 \%$ for SNP 3, 52.4\% for SNP 5, and $57.3 \%$ for SNP 6. Lazar et al. [28] examined OCT3 gene polymorphism in 100 Caucasian individuals and found the frequency of the $\mathrm{M}$ allele to be $64 \%$ for SNP1 and $63.5 \%$ for SNP 7. Our findings for M allele frequency in the control group are $54.7 \%$ for SNP 1 and $55.3 \%$ for SNP 7. Therefore, the M allele frequencies we found in the US Caucasian population are relatively consistent with frequencies found in different geographic and ethnic groups.

SNPs $1,2,3$, and 7 have been found to be associated with reduced OCT3 mRNA levels in the human liver [29]. However, at this point it is not known whether this translates to reduced OCT3 mRNA levels or altered OCT3 function in the brain for these particular SNPs. A recent study by Sakata et al. [30] analyzed the functional properties of five OCT3 SNPs resulting in the amino acid changes using a transient expression system. The study found decreased OCT3 uptake capacity for three of those five SNPs tested. However, the minor allele frequency for these three SNPs is extremely low (less than 1\%), which leads us not to pursue evaluation of these three SNPs at this time. Obviously, further studies using these and additional SNPs are needed.

Several limitations of the present study should be noted. First, with the sample size of 82 subjects in the depressed group and 75 subjects in the control group, it is possible to detect a difference of $20 \%$ in each allele frequency, but not differences lower than $20 \%$. Second, our study did not include the data on the severity of depression in the depressed group or the information on existence of other medical comorbidities in either group.

Recently, OCT3 has received considerable attention in regard to its role in the regulation of monoamine neurotransmission under both basal conditions [31] and during stress $[32,33]$. In addition, studies using animal models of depression demonstrated antidepressant effects of both pharmacological [34] and antisense blockade [35] of OCT3. As treatment-resistant depression continues to present a significant clinical problem [36], and there is an ongoing search for new antidepressant drugs, these data support the notion of OCT3 as a potential new antidepressant therapy target $[31,37]$.

In conclusion, this study is, to our knowledge, the first to compare the allele and genotype frequencies of seven OCT3 gene SNPs between a US Caucasian depressed population and a control group of nondepressed subjects. No significant difference was found between these two groups for any of the SNPs examined. However, further studies are needed to verify these findings.

\section{Acknowledgments}

The authors thank Dr. J. Hammill for help with real-time PCR runs and Dr. A. Harralson for helpful discussions. This paper was supported by the Bernard J. Dunn School of Pharmacy, Shenandoah University Faculty Development Grant to Nina Hengen.

\section{References}

[1] O. Berton and E. J. Nestler, "New approaches to antidepressant drug discovery: beyond monoamines," Nature Reviews Neuroscience, vol. 7, no. 2, pp. 137-151, 2006. 
[2] B. Haenisch and H. Bönisch, "Depression and antidepressants: insights from knockout of dopamine, serotonin or noradrenaline re-uptake transporters," Pharmacology and Therapeutics, vol. 129, no. 3, pp. 352-368, 2011.

[3] A. Frazer, "Pharmacology of antidepressants," Journal of Clinical Psychopharmacology, vol. 17, supplement 1, no. 2, pp. 2S-18S, 1997.

[4] J. Masson, C. Sagné, M. Hamon, and S. El Mestikawy, "Neurotransmitter transporters in the central nervous system," Pharmacological Reviews, vol. 51, no. 3, pp. 439-464, 1999.

[5] S. G. Amara and M. J. Kuhar, "Neurotransmitter transporters: recent progress," Annual Review of Neuroscience, vol. 16, pp. 73-93, 1993.

[6] G. Rudnick and J. Clark, "From synapse to vesicle: the reuptake and storage of biogenic amine neurotransmitters," Biochimica et Biophysica Acta, vol. 1144, no. 3, pp. 249-263, 1993.

[7] H. Koepsell, K. Lips, and C. Volk, "Polyspecific organic cation transporters: structure, function, physiological roles, and biopharmaceutical implications," Pharmaceutical Research, vol. 24, no. 7, pp. 1227-1251, 2007.

[8] A. Amphoux, V. Vialou, E. Drescher et al., "Differential pharmacological in vitro properties of organic cation transporters and regional distribution in rat brain," Neuropharmacology, vol. 50, no. 8, pp. 941-952, 2006.

[9] V. Vialou, L. Balasse, J. Callebert, J. M. Launay, B. Giros, and S. Gautron, "Altered aminergic neurotransmission in the brain of organic cation transporter 3-deficient mice," Journal of Neurochemistry, vol. 106, no. 3, pp. 1471-1482, 2008.

[10] P. J. Gasser, M. Orchinik, I. Raju, and C. A. Lowry, "Distribution of organic cation transporter 3, a corticosteronesensitive monoamine transporter, in the rat brain," Journal of Comparative Neurology, vol. 512, no. 4, pp. 529-555, 2009.

[11] N. Feng, B. Mo, P. L. Johnson, M. Orchinik, C. A. Lowry, and K. J. Renner, "Local inhibition of organic cation transporters increases extracellular serotonin in the medial hypothalamus," Brain Research, vol. 1063, no. 1, pp. 69-76, 2005.

[12] P. J. Gasser, C. A. Lowry, and M. Orchinik, "Corticosteronesensitive monoamine transport in the rat dorsomedial hypothalamus: potential role for organic cation transporter 3 in stress-induced modulation of monoaminergic neurotransmission," Journal of Neuroscience, vol. 26, no. 34, pp. 87588766, 2006.

[13] E. J. Nestler, M. Barrot, R. J. DiLeone, A. J. Eisch, S. J. Gold, and L. M. Monteggia, "Neurobiology of depression," Neuron, vol. 34, no. 1, pp. 13-25, 2002.

[14] X. Wu, R. Kekuda, W. Huang et al., "Identity of the organic cation transporter OCT3 as the extraneuronal monoamine transporter (uptake2) and evidence for the expression of the transporter in the brain," Journal of Biological Chemistry, vol. 273, no. 49, pp. 32776-32786, 1998.

[15] M. Hayer-Zillgen, M. Brüss, and H. Bönisch, "Expression and pharmacological profile of the human organic cation transporters hOCT1, hOCT2 and hOCT3," British Journal of Pharmacology, vol. 136, no. 6, pp. 829-836, 2002.

[16] J. E. Hill, K. Makky, L. Shrestha, C. J. Hillard, and P. J. Gasser, "Natural and synthetic corticosteroids inhibit uptake2mediated transport in CNS neurons," Physiology and Behavior, vol. 104, no. 2, pp. 306-311, 2010.

[17] M. Cui, R. Aras, W. V. Christian et al., "The organic cation transporter-3 is a pivotal modulator of neurodegeneration in the nigrostriatal dopaminergic pathway," Proceedings of the National Academy of Sciences of the United States of America, vol. 106, no. 19, pp. 8043-8048, 2009.
[18] T. Wultsch, G. Grimberg, A. Schmitt et al., "Decreased anxiety in mice lacking the organic cation transporter 3," Journal of Neural Transmission, vol. 116, no. 6, pp. 689-697, 2009.

[19] D. L. Murphy, A. Lerner, G. Rudnick, and K. P. Lesch, "Serotonin transporter: gene, genetic disorders, and pharmacogenetics," Molecular Interventions, vol. 4, no. 2, pp. 109-123, 2004.

[20] K. P. Lesch and L. Gutknecht, "Pharmacogenetics of the serotonin transporter," Progress in Neuro-Psychopharmacology and Biological Psychiatry, vol. 29, no. 6, pp. 1062-1073, 2005.

[21] P. Zill, R. Engel, T. C. Baghai et al., "Identification of a naturally occurring polymorphism in the promoter region of the norepinephrine transporter and analysis in major depression," Neuropsychopharmacology, vol. 26, no. 4, pp. 489493, 2002.

[22] S. H. Ryu, S. H. Lee, H. J. Lee et al., "Association between norepinephrine transporter gene polymorphism and major depression," Neuropsychobiology, vol. 49, no. 4, pp. 174-177, 2004.

[23] K. Inoue, K. Itoh, K. Yoshida, T. Shimizu, and T. Suzuki, "Positive association between $\mathrm{T}-182 \mathrm{C}$ polymorphism in the norepinephrine transporter gene and susceptibility to major depressive disorder in a Japanese population," Neuropsychobiology, vol. 50, no. 4, pp. 301-304, 2004.

[24] D. A. Trégouët, I. R. König, J. Erdmann et al., "Genome-wide haplotype association study identifies the SLC22A3-LPAL2LPA gene cluster as a risk locus for coronary artery disease," Nature Genetics, vol. 41, no. 3, pp. 283-285, 2009.

[25] R. Sallinen, M. A. Kaunisto, C. Forsblom et al., "Association of the SLC22A1, SLC22A2, and SLC22A3 genes encoding organic cation transporters with diabetic nephropathy and hypertension," Annals of Medicine, vol. 42, no. 4, pp. 296-304, 2010.

[26] N. Aoyama, N. Takahashi, K. Kitaichi et al., "Association between gene polymorphisms of SLC22A3 and methamphetamine use disorder," Alcoholism, vol. 30, no. 10, pp. 16441649, 2006.

[27] A. Lazar, S. Walitza, A. Jetter et al., "Novel mutations of the extraneuronal monoamine transporter gene in children and adolescents with obsessive-compulsive disorder," International Journal of Neuropsychopharmacology, vol. 11, no. 1, pp. 35-48, 2008.

[28] A. Lazar, D. Gründemann, R. Berkels, D. Taubert, T. Zimmermann, and E. Schömig, "Genetic variability of the extraneuronal monoamine transporter EMT (SLC22A3)," Journal of Human Genetics, vol. 48, no. 5, pp. 226-230, 2003.

[29] A. T. Nies, H. Koepsell, S. Winter et al., "Expression of organic cation transporters OCT1 (SLC22A1) and OCT3 (SLC22A3) is affected by genetic factors and cholestasis in human liver," Hepatology, vol. 50, no. 4, pp. 1227-1240, 2009.

[30] T. Sakata, N. Anzai, T. Kimura et al., "Functional analysis of human organic cation transporter OCT3 (SLC22A3) polymorphisms," Journal of Pharmacological Sciences, vol. 113, no. 3, pp. 263-266, 2010.

[31] L. C. Daws, "Unfaithful neurotransmitter transporters: focus on serotonin uptake and implications for antidepressant efficacy," Pharmacology and Therapeutics, vol. 121, no. 1, pp. 89-99, 2009.

[32] N. Baganz, R. Horton, K. Martin, A. Holmes, and L. C. Daws, "Repeated swim impairs serotonin clearance via a corticosterone-sensitive mechanism: organic cation transporter 3, the smoking gun," Journal of Neuroscience, vol. 30, no. 45, pp. 15185-15195, 2010. 
[33] N. Feng, C. A. Lowry, J. L. Lukkes, M. Orchinik, G. L. Forster, and K. J. Renner, "Organic cation transporter inhibition increases medial hypothalamic serotonin under basal conditions and during mild restraint," Brain Research, vol. 1326, pp. 105-113, 2010.

[34] N. L. Baganz, R. E. Horton, A. S. Calderon et al., "Organic cation transporter 3: keeping the brake on extracellular serotonin in serotonin-transporter-deficient mice," Proceedings of the National Academy of Sciences of the United States of America, vol. 105, no. 48, pp. 18976-18981, 2008.

[35] K. Kitaichi, M. Fukuda, H. Nakayama et al., "Behavioral changes following antisense oligonucleotide-induced reduction of organic cation transporter-3 in mice," Neuroscience Letters, vol. 382, no. 1-2, pp. 195-200, 2005.

[36] M. Fava, "Diagnosis and definition of treatment-resistant depression," Biological Psychiatry, vol. 53, no. 8, pp. 649-659, 2003.

[37] J. J. Schildkraut and J. J. Mooney, "Toward a rapidly acting antidepressant: the normetanephrine and extraneuronal monoamine transporter (Uptake 2) hypothesis," American Journal of Psychiatry, vol. 161, no. 5, pp. 909-911, 2004. 

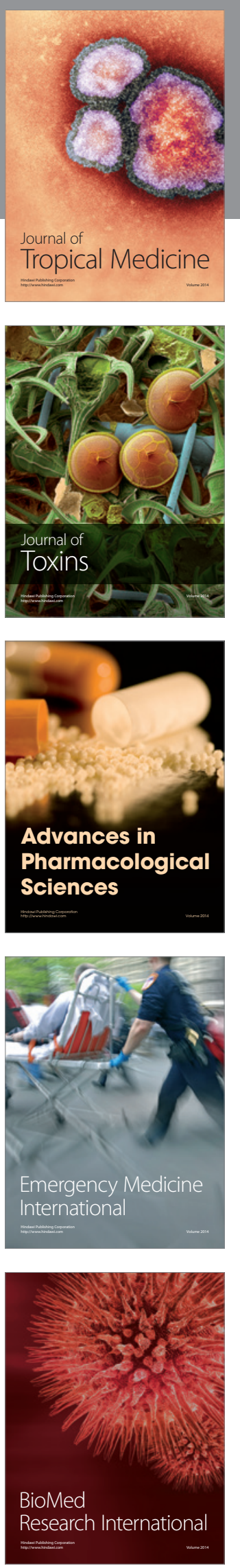
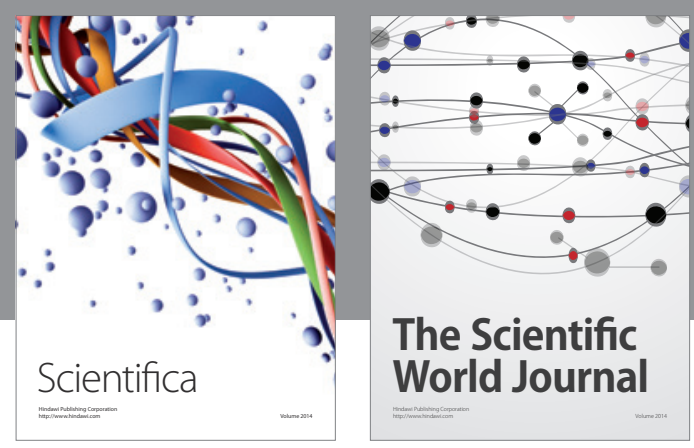

The Scientific World Journal
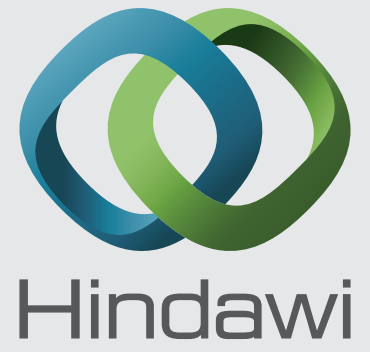

Submit your manuscripts at

http://www.hindawi.com
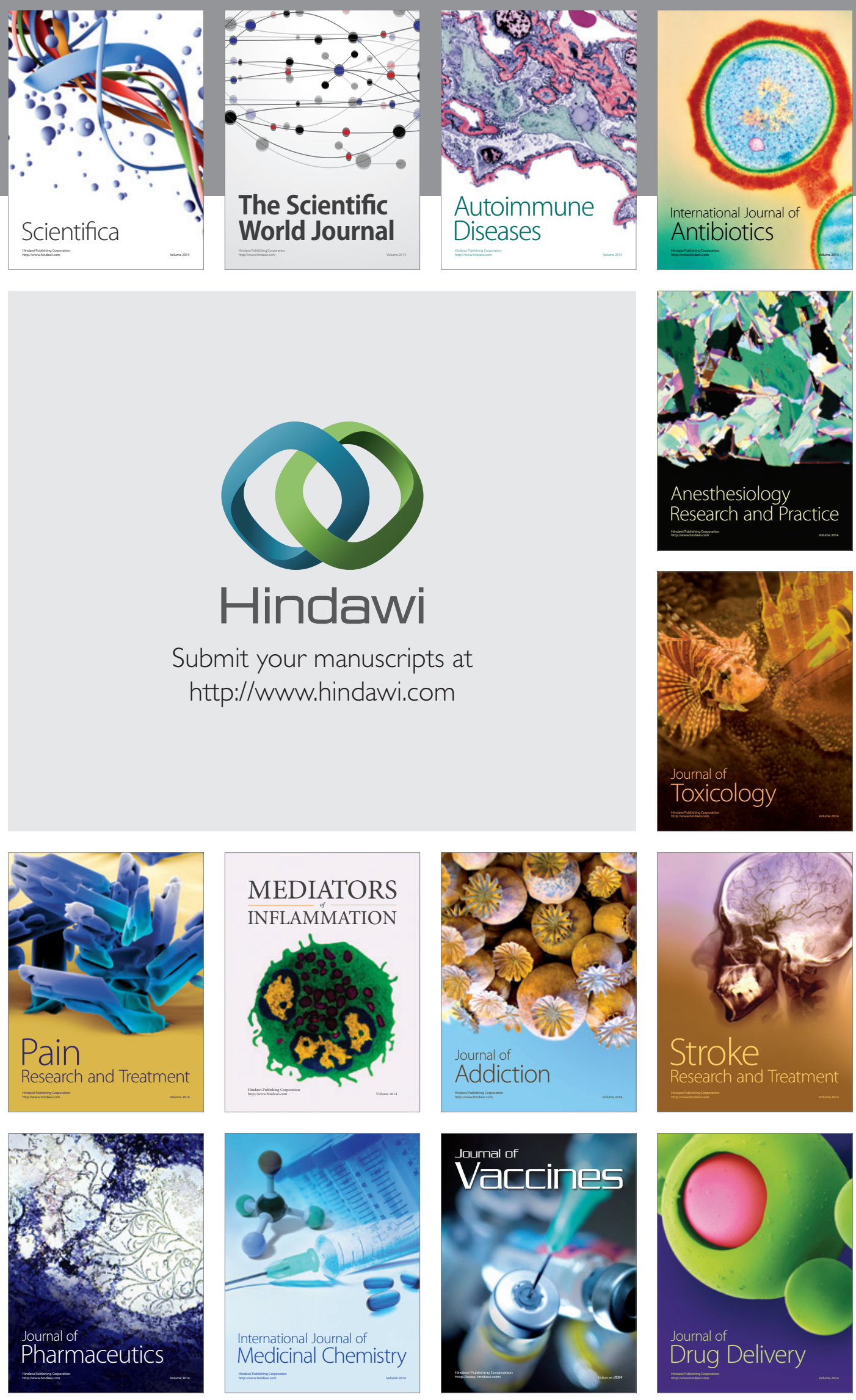